\title{
A taxonomy of cross layer routing metrics for wireless mesh networks
}

\author{
Md Asri Bin Ngadi ${ }^{1}$, Saqib Ali ${ }^{*}$, Abdul Hanan Abdullah ${ }^{1}$ and Rashid Hafeez Khokhar ${ }^{2}$
}

\begin{abstract}
Multi-hop, multi-channel, and multi-radio wireless mesh networks (WMNs) are emerging as promising field of wireless technology with self-organizing and self-healing features for internet and real time applications, i.e., VolP and Video over IP. Interoperability feature of WMNs have made them to integrate easily with other network technologies like wired networks, WiFi, WiMax, MANETs, and cellular networks. WMNs are gaining popularity due to their high network throughput which highly depends on the routing procedures. Routing algorithms like optimized link state routing protocol and dynamic source routing make efficient routing decisions on the basis of routing metrics which actually predict the cost of link quality. Most of the routing protocols and routing metrics implemented in WMNs are actually designed for mobile ad hoc networks (MANETs). Since WMNs have different characteristics and limitations as compared to MANETs, so the routing metrics design for MANETs do not perform well in WMNs. Furthermore, quality of service (QoS) and throughput of the network in WMNs can be enhanced by using cross layer routing approach and by deploying multi-channel multi-radio (MCMR) scenarios in each relay node. This article discusses a design taxonomy, limitations and qualitative comparison of existing routing metrics for QoS in MCMR WMNs with respect to routing parameters, i.e., transmission rate, inter-flow interference, intra-flow interference, congestion, and channel diversity. Moreover, our taxonomy also opens the door up for new research areas in the design of cross layer routing metrics for MCMR radio WMNs for high throughput IP connectivity.
\end{abstract}

\section{Introduction}

Wireless mesh networks (WMNs) are cooperative multihop, self-organizing, self-configuring, self-healing, and fault tolerant communication networks. The use of cooperative multi hopping technique helps the wireless nodes to route between node to node, node to multi-hop destination node, and node to base station, i.e., internet backhaul. WMNs provide cooperate low up-front cost solution for high speed internet connectivity in urban and wilderness areas as compare to other technologies like wired and optical networks [1]. WMNs inherit many features of wired networks, static wireless networks, and wireless ad hoc networks which are necessary in order to develop a multi-hop self-organizing and self-healing solution for last mile IP connectivity in community networks as shown in Figure 1. These inherited features make WMNs richer in resources as compared to other stand

\footnotetext{
*Correspondence: asaqib2@live.utm.my

${ }^{1}$ Faculty of Computer Science and Information Systems, Universiti Teknologi Malaysia, 81310 Skudai, Johor, Malaysia

Full list of author information is available at the end of the article
}

alone networks. Use of MCMR techniques in WMNs has further increased its throughput capacity, reliability, and robustness as each wireless interface is linked with its own separate medium access control (MAC) and physical layer [2]. Furthermore, WMNs can be integrated with other network technologies such as wired networks, wirelessfidelity (Wi-Fi) [3], mobile ad hoc networks (MANETs) [4], wireless sensor networks (WSNs) [5], vehicular ad hoc networks (VANETs) [6], and Worldwide interoperability for microwave access (WiMax) [7] through the bridging abilities of the mesh routers [8].

Routing metrics predict the cost of the route calculated by the routing protocols. They provide quantifiable values that can be used to judge the cost or efficiency of a route. Since WMNs inherit many features of MANETs, routing protocols and routing metrics developed for MANETs can be set up for WMNs. Present framework of IEEE 802.11s deploy an ad hoc on-demand distance vector (AODV) algorithm as a major building block for routing in WMNs [9]. Power requirements and mobility features of WMNs are very different from ad hoc networks. Mesh

\section{是 Springer}

(c) 2012 Bin Ngadi et al: licensee Springer. This is an Open Access article distributed under the terms of the Creative Commons Attribution License (http://creativecommons.org/licenses/by/2.0), which permits unrestricted use, distribution, and reproduction in any medium, provided the original work is properly cited. 


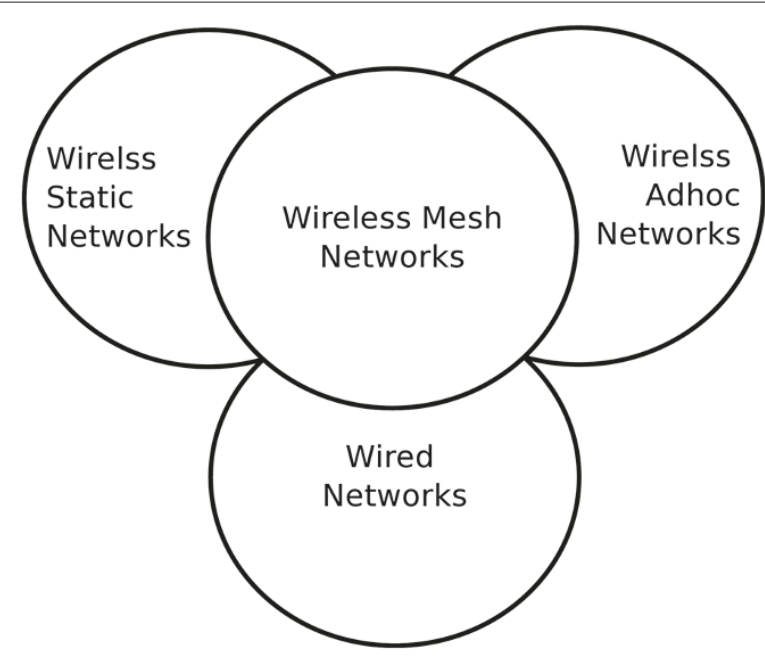

Figure 1 Hybrid nature of WMN.

routers possess minimal mobility with no power constraints where as mesh clients are mobile with limited power. Therefore, routing metrics designed for ad hoc networks does not perform proficiently in WMNs [10]. Moreover, WMNs support MCMR technologies which provide each node with multiple radios for improving the capacity of the networks, stipulate efficient routing protocols and metrics for WMNs [11]. Also cross layer communication between network layer and MAC layer or physical layer has increased the performance of routing protocols in MCMR WMNs especially in real time and multi-cast applications [12]. Thus design of routing protocols and routing metrics play a critical role in order to find out the efficient route to the destination in a network [13]. Consequently this study is focused on the taxonomy, limitations, recent challenges and future perspective in the design and development of cross layer routing metrics for MCMR WMNs. Design taxonomy of the routing metrics will be based on the parameters classified as basic (transmission rate, packet loss ratio, delay), interference aware (received signal strength (RSSI), bit error rate, frame error rate, signal to noise ratio (SNR)), and load aware (number of flows, queue size). Limitations of each metric is discussed in detail with respect to real time applications like VoIP, Video over IP and multi-casting in MCMR WMNs. Qualitative comparison, recent challenges, and future perspective in the design and development of cross layer routing metrics are also summarized.

The rest of the article is organized as follows. The components and architecture of WMNs are discussed in Section 2. Characteristics and application scenarios of WMNs are elaborated in Section 3. Cross layer routing in MCMR WMN is explained in Section 4. Design taxonomy, characteristics and limitations of existing routing metrics are discussed in Section 5. Qualitative comparison and summary of the routing metrics is elaborated in Section 6. Finally, the conclusions and future perspective of this research is presented in Section 7.

\section{Components and network architecture of WMNs}

WMNs work as backhaul networks which provide broad band services to homes, offices, security systems, transportation systems. WMNs also provide interoperability with several wireless and wired networks. Thus they provide a bench mark for community multi-hop ad hoc networks [14]. Components of WMNs are explained as follows:

\subsection{Components of WMNs}

There are three main components of WMNs such as mesh gateway routers, mesh routers, and mesh clients. Mesh gateway routers provide internet access to the network, mesh routers develop the main backbone of the wireless network where as mesh clients serve as a end user devices in the network. These components are explained in detail as follows:

Mesh gateway routers act as a network backhaul for communality networks with bridging and routing functionality which allow them to incorporate with different wired and wireless networks like Ethernet, mobile ad hoc wireless networks (MANETS), wireless sensor networks (WSNs), Wi-Fi, VANETS, and WiMax [15].

Mesh routers provide multi-hop routing between mesh gateways and mesh clients with self organizing and self healing features. Minimum mobility with no power constraints features help them to form the backbone of the wireless network. Mesh routers are also outfitted with multi channel single radio or MCMR devices to further enhance the capacity of the network.

Mesh clients are the end user devices in WMNs with high mobility and power constraint features, i.e., laptops, IP phones, personal digital assistant (PDA), and pocket PC. Mesh clients are normally provided with single radio devices and may or may not have routing functionality depending on the architecture of the network.

Components of WMNs are shown in Figure 2. Wireless mesh routers which are in the middle of the figure, act as a relay nodes and form the backbone of the network. Solid line indicate the wired link between mesh gateway router and wireless mesh router for internet connectivity. Dotted lines indicate the wireless connectivity among wireless mesh routers, user end devices (PCs, Laptops, PDAs, Pocket PCs, IP Phones, Wi-Fi Printers), and other wireless networks (Mobile Cellular Networks, WiMAX, VANETs).

\subsection{Architecture of WMNs}

WMN is comprised of three main type of architecture such as client WMNs, hierarchical WMNs, and hybrid 


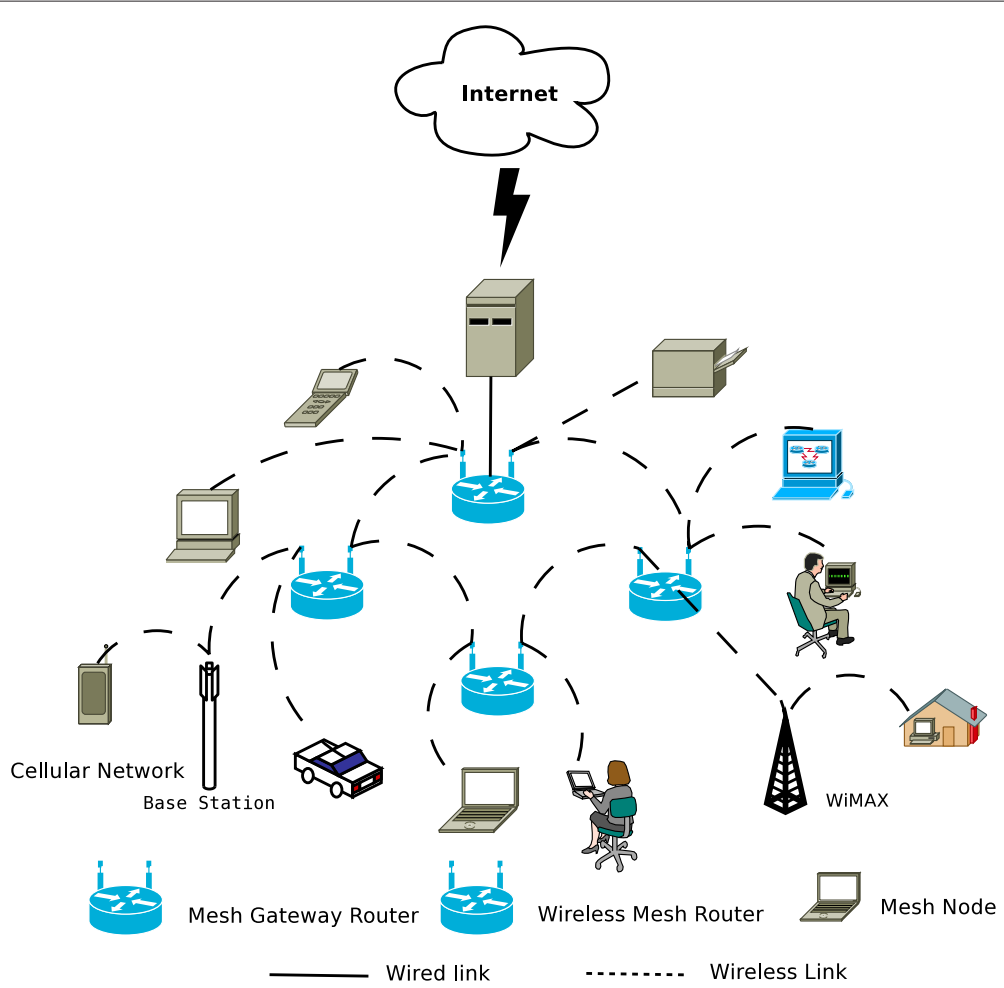

Figure 2 Components and architecture of mesh network.

WMNs [2]. Characteristics of each architecture is explained as follows:

Client WMN provides peer-to-peer networking facility between the client nodes. Client nodes are normally single radio devices with optional routing capability depending on the end user requirements. Client WMNs are very simple to design but scalability and limited resource allocation are the critical issues which may cause throughput degradation in the network.

Hierarchical WMNs consist of a hierarchy in which mesh gateway routers are at the top with bridging functionality for backhaul internet connectivity, mesh routers are in the middle with self configuring and self healing functionality to act as a network backbone and mesh clients are at the bottom to serve as an end user devices as shown in the Figure 2.

Hybrid WMNs consist of both hierarchical and client architectures. Mesh routers are equipped with the bridging functionality in order to integrate with other networks like wired networks, WiFi, MANETs and VANETs as shown in the Figure 2. multi-hop cellular networks is an example of hybrid WMNs [16].

\section{Characteristics and application scenarios of WMNs}

Multi hop, self-organizing and self-healing WMNs are popular in real time applications for there increased throughput and reliability as compared to other networks like WiFi and MANETs. Characteristic of WMNs are summarized under the following headings:

\subsection{Low up-front deployment cost}

Installation cost of $802.11 \mathrm{WiFi}$ frameworks is quite high as compared to WMNs since cable connectivity to the network backbone, is required by each access point (AP) for internet access. Moreover, transmission range of 802.11 WiFi framework is very limited and often cause the problem of dead zones where as WMNs do not need any cabling structure to internet backhaul and dead zones are easily eliminated by changing the position of the mesh router. As a result coverage area in WMNs can be extended easily and more quickly because of ad hoc nature as compared to 802.11 based APs.

\subsection{Reliability and robustness}

WMNs are multi hop wireless networks with redundant paths between source and the destination. Therefore chance of single point of failure due to hardware failure, path failure, obstacle or power outage is eliminated. Furthermore, multiple links between the nodes also facilitate in avoiding the congested and the bottleneck links present in the network. Thus the communication in the network becomes more reliable and robust in nature [17]. 


\subsection{Multi-channels multi-radios}

Today, WMNs support MCMR scenarios which has further increased the overall throughput of the network. Mesh routers are equipped with two or more radios as each radio has its own MAC and Physical layer. Non overlapping frequency channels present in a single radio are further used to increase the throughput of the network [18]. Multiple radios with multiple channels are available between the source and destination for communication enhancement [19]. In IEEE 802.11, for example $2.4-2.4835 \mathrm{GHz}$ frequency band has been alienated into 13 channels each having the width of $22 \mathrm{MHz}$. It has only three non-overlapping channels as shown by the solid green, red and blue lines in the Figure 3. Figure 4 indicates the protocol stack of IEEE 802.11s mesh gateway router with bridging functionality, mesh router with MCMR technology and mech client with single radio functionality, respectively, [9]. MCMR scenario in wireless mesh router is shown in Figure $4 \mathrm{~b}$, in which mesh router is equipped with two radios: Radio 1 and Radio 2. Each radio has its own MAC and Physical layer. Communication is carried out by three non overlapping channels $\mathrm{C} 1, \mathrm{C} 2$, and $\mathrm{C} 3$ in $2.4 \mathrm{GHz}$ frequency band, which has increase the overall throughput of the router up to six times as compared to single channel single radio router. Multi-radio technology also assist in the interoperability of WMNs with other networks like WiFi, WiMAX, wireless sensor networks and cellular networks.

\subsection{COTS products}

Common off-the-shelf (COTS) is a technology which is ready-made and easily available to the general public. Motivation for using COTS is to facilitate the development and minimize the cost of the product. COTS products include computer software, hardware systems or free software. Deployment of WMN is quite easy as compared to other wireless networks because most of the time network backbone consists of COTS products which are cheap and easily available. For example mesh routers and mesh gateways can be deployed by using normal personal computers.

\subsection{Application scenarios of WMNs}

WMNs broadband services are playing an effective role in the home, office, enterprise and community networks. Especially, multi-casting feature of WMN support Video over IP in community networks with high level of quality of service (QoS) by making delay variation and packet loss ratio to minimum [20]. Public transportation system, public safety surveillance system, medical health system in hospitals, and Voice over IP (VoIP) or Internet telephony in community networks are making effective use of WMNs [21]. During rescue operation due to natural disasters (floods, earthquakes or landslides), WMNs are helping by developing a peer to peer communications at anytime and anywhere between the group of people. US department of defense is also taking benefits from WMNs in the battlefields due to its self-organizing, self-healing, and ad hoc nature [22].

\section{Cross layer routing approach in MCMR WMNs}

Recently IEEE 802.11 based routing in WMNs has been actively explored to provide high-speed, reliable, and robust IP connectivity in societal networks. Routing protocols in WMNs can be proactive, i.e., optimized link state routing protocols (OLSR) [23], modified optimized link state routing [24], Highly Dynamic Destination Sequenced Distance vector routing protocol [25], multipath destination sequenced distance vector [26], reactive, i.e., AODV routing protocol [27], Multicast ad-hoc ondemand distance vector [28], dynamic source routing [29], multi-radio link-quality source routing [11], and hybrid, i.e., hybrid wireless mesh protocol [30], temporallyordered routing algorithm [31]. General taxonomy of routing protocols for multi-hop wireless networks is shown in Figure 5. Cross layer routing protocol or metrics design is actually the collaboration across the borders, i.e., sharing of information between different layers such as network layer, MAC layer and physical layer [32]. The concept of cross-layer design is helpful in improving the overall network performance. Basic routing metrics are developed on the basis of the shortest path metric (hop count) which actually does not predict the true channel

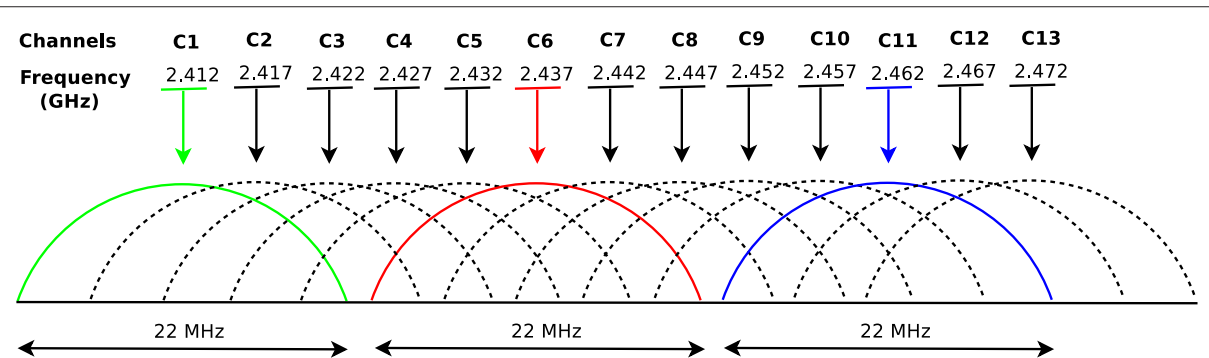

Figure 3 Three non overlapping channels in IEEE $802.11 \mathrm{~b}$ standard. 


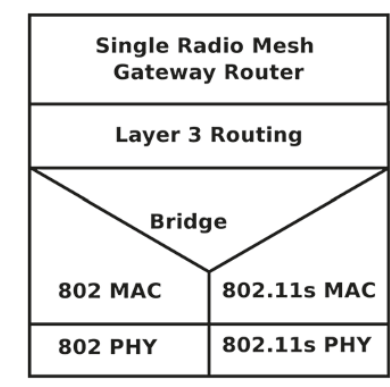

\begin{tabular}{|c|c|c|c|c|c|}
\hline \multicolumn{5}{|c|}{$\begin{array}{c}\text { Multi Radio Mesh } \\
\text { Router }\end{array}$} \\
\hline \multicolumn{2}{|c|}{ Radio 1 } & \multicolumn{3}{|c|}{ Radio 2 } \\
\hline \multicolumn{2}{|c|}{ MAC 802.11s } & \multicolumn{2}{|c|}{ MAC 802.11s } \\
\hline \multicolumn{2}{|c|}{ PHY 802.11s } & \multicolumn{2}{|c|}{ PHY 802.11s } \\
\hline C1 & C2 & C3 & C1 & C2 & C3 \\
\hline
\end{tabular}

Figure 4 Protocol stack of IEEE $\mathbf{8 0 2 . 1 1}$ s. (a) static mesh gateway router (b) mesh client with single radio. characteristic effectively, especially in terms of interference and traffic congestion [33,34]. The main idea behind cross layer routing is to provide the characteristics of Network layer, Media Access Control layer and Physical layer to the routing metric for capturing the true link quality in a dynamic way [35]. Furthermore, in MCMR WMNs congestion and interference between channel and radios have a critical effect on the overall throughput and efficiency of the network. Thus cross layer routing metric design is mandatory in MCMR mesh networks. Table 1 summarized the characteristics of mesh gateway router, mesh routers, and mesh client. Mesh gateway routers are static in nature with no power limitations, mesh routers are normally static with minimum mobility where as mesh clients are mostly mobile with limited power. Theses characteristic have a critical effect on the design of cross layer routing metric in MCMR WMNs [17].

\subsection{Design parameters of cross layer routing metrics in MCMR WMNs}

Routing protocols use routing metrics which actually predict the weight of the link or the path in order to make efficient routing decisions. Parameters related to the design of cross layer routing metrics in MCMR WMNs can be categorized as Basic, Load aware, Interference aware, and QoS. In spite of these, selected path or route must be Isotonic in nature in order to carry out loop free routing. Furthermore, asymmetry of the wireless links (transmission behavior of wireless link is different in different direction), and route stability parameters are very critical in the design of efficient cross layer routing metric in MCMR WMNs. Theses parameters are explained in detail as follows:

Basic, load, interference, and QoS parameters related to physical layer, MAC layer, and network layer of multi hop wireless networks are very multifaceted in nature as compared to wired networks because of shared wireless medium. Basic (path length, packet loss ratio, delay), load (queue size, no. of flows), interference (intra-flow interference, inter-flow interference), and QoS (link capacity, overall throughput, power utilization) parameters related to design of cross layer routing metrics are summarized in the Table 2 which not only predict the link quality of the network but also facilitate in selecting the efficient route in multi-hop wireless network [36-43].

Isotonic aware property of the routing metric is an important design parameter for selecting optimum weight paths and to avoid routing loops. Isotonic property of the routing metric is defined as, the order of weights of two paths is preserved if they are connected to a common third path. Isotonic property of the routing metric must be followed to calculate the optimum paths using Dijkstra's algorithms in multi hop routing scenarios [44].

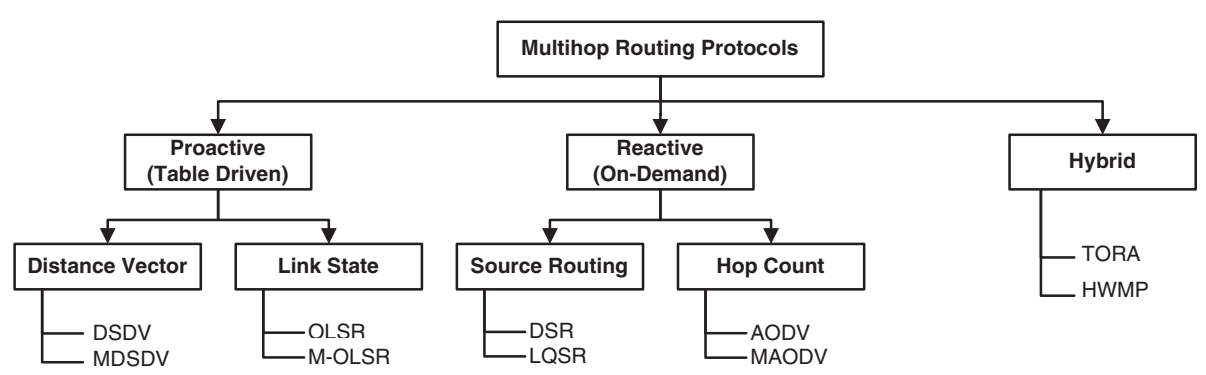

Figure 5 Routing protocols for multi-hop wireless networks. 
Table 1 Routing characteristics summary

\begin{tabular}{|c|c|c|c|}
\hline Characteristics & $\begin{array}{l}\text { Gateway } \\
\text { router }\end{array}$ & Mesh router & Mesh client \\
\hline $\begin{array}{l}\text { Network } \\
\text { topology }\end{array}$ & Always static & Normally static & Mostly mobile \\
\hline $\begin{array}{l}\text { Interfaces per } \\
\text { node }\end{array}$ & One or more & One or more & Normally one \\
\hline Mobility model & Static in nature & $\begin{array}{l}\text { Minimum } \\
\text { mobility }\end{array}$ & Highly mobile \\
\hline $\begin{array}{l}\text { Interference } \\
\text { intensity }\end{array}$ & Low intensity & High intensity & Low intensity \\
\hline Power limitations & No limitations & No limitations & Limited power \\
\hline Channel diversity & Present & Present & Not present \\
\hline
\end{tabular}

Asymmetry of wireless Links actually define the propagation behavior of links which is quite different in different directions as compared to wired links. Disseminate packets normally send from a source node may successfully be received at the destination node but the connection may failed when the destination node want to send replay packets back to source node. This criteria is known as asymmetry of wireless link [45]. Asymmetry of the wireless links may reach up to 5 to $15 \%$ as mentioned by Ganesan et al. [46]. Hence asymmetry of link must also be taken in to account while developing the routing metrics for WMNs.

Route stability parameters effect the overall throughput of the network. Since the overall performance of the network is highly dependent on the route stability parameters which actually minimize the fluctuation of the route after being declared as an efficient one. Frequent path oscillations results in the poor network performance because these frequent changes in path weight cause an increase in the number of route update packets. Route stability mechanism in wireless networks can be achieved by setting a limit of $10 \%$ throughput increase over the route which is currently being used by the routing protocol [47].
Design parameters shown in Table 2 reside at different level of the network. Design of routing metric may consist of one or more parameters. However, it is a very challenging research problem to design a MCMR routing metric so that it will capture all above mentioned parameters [48]. On the basis of above discussion, taxonomy of available routing metrics for WMNs is explained in the following section.

\section{Cross layer routing metrics for MCMR WMNs}

High link losses, asymmetric link, and MCMR functionality in WMNs have made the design of routing metric quite challenging. However quite a good number of routing metrics are designed in the recent years for WMNs. This section will scrutinize the existing routing metrics with its definition and limitations in MCMR WMNs on the basis of parameters explained in Section 4.

\subsection{Expected transmission count}

Expected transmission count (ETX) is defined as the number of expected transmission plus retransmissions required to successfully deliver a packet over a wireless link [49]. If forward delivery ratio $d_{\text {fwd }}$, i.e., probability that the packet successfully received at destination node and reverse delivery ratio $d_{r v s}$, i.e., probability that acknowledgment of the packet successfully received at source node, then ETX of the link is calculated as follows:

$$
\mathrm{ETX}=\frac{1}{d_{\mathrm{fwd}} \cdot d_{\mathrm{rvs}}}
$$

ETX metric has significantly improved performance over minimum hop count routing metric as shown by the test bed results [50]. ETX develop its design foundation on delivery ratios which truly effects the throughput as compared to minimum hop count metric. Furthermore, ETX take account of asymmetry of links in a duplex manner by considering the loss ratios. The utilization of the spectrum

Table 2 Parameters for cross layer routing metric

\begin{tabular}{llll}
\hline Classification & Parameters & Monitoring & Measurement \\
\hline \multirow{2}{*}{ Basic } & Path length & Active probing & Per flow \\
& Packet loss ratio & Active probing & Per link flow \\
Load aware & Delay & Active probing & Per link \\
& Queue size & Passive monitoring & Per link \\
Interference aware & No. of flows & Passive monitoring & Per flow \\
& Intra-flow interference & Channel diversity & Per node \\
QoS & Inter-flow interference & Signal strength & Per link \\
& Link capacity & Active probing & Per network \\
\hline
\end{tabular}


is also minimized by ETX which is helpful in increasing the capacity of the network.

ETX was actually designed for single-channel singleradio multi-hop wireless networks so it does not capture the channel diversity in MCMR multi-hop wireless networks. The design of ETX only predict about the inter flow interference by considering the loss ratios in a static manner but it does not have any information about the intra-flow interference faced by the links. In addition, active probing technique fail to predict the queuing delay in the network and without load balancing mechanism ETX matrix may lead the traffic to bottleneck routes in the networks. Active probing technique incorporated by this metric to capture the loss ratio may result in underestimation or overestimation of losses because data packets of IEEE 802.11 real transmission are of different sizes as compared to probe packets of same size, i.e., 134 bytes [49]. ETX is based on average or mean loss ratio where as in WMNs burst losses exists which does not make off well by this routing metric [51]. In addition, ETX does not take account the option that different communication links may possesses different transmission rates which has a critical effect on the network throughput.

As discuss earlier, the poor performance of ETX is mainly due to the assumption that channel conditions are static in nature, i.e., average or mean packet loss ratio where as channel conditions in wireless networks varies dynamically from time to time. To overcome the drawbacks of ETX, modified expected number of transmission (mETX) and effective number of transmission (ENT) are designed on the basis of link variance in order to make ETX an quality aware routing metric [51]. mETX is calculated as follows:

$$
\operatorname{mETX}=\exp \left(\mu+\frac{1}{2} \sigma^{2}\right)
$$

where $\mu$ is average or mean packet loss ratio and $\sigma^{2}$ is variance of packet loss ratio.

ENT is calculated as follow:

$$
\mathrm{ENT}=\exp \left(\mu+\frac{1}{2} \delta \sigma^{2}\right)
$$

where $\delta$ is the strictness of the loss rate requirement.

Although mETX and ENT are improved form of ETX but they still failed to capture the link quality in terms of inter-flow and intra-flow interferences of the route [13]. Furthermore, they compute the losses on the basis of bit error rate which is quite infeasible due to its complex verification mechanism and MAC layer error packet drop mechanism.

\subsection{Expected transmission time}

IEEE 802.11 MAC layer protocols have multi rate transmission ability which has increased the throughput of the wireless networks significantly [52]. EXT design does not mention that different communication links may have different transmission rates. EXT was developed only by considering the average channel conditions. To solve the problems of ETX, Draves et al. [11] designed expected transmission time (ETT) which has significantly enhanced the performance of ETX by measuring the transmission rate of each individual link. ETT is defined as follows:

$$
\begin{aligned}
& \mathrm{ETT}=\sum_{j}^{n} \mathrm{ETT}_{j}=\sum_{j}^{n} \operatorname{ETX}_{j} \times\left(\frac{S}{B_{j}}\right) \\
& \mathrm{ETT}=\sum_{j}^{n} \mathrm{ETT}_{j}=\sum_{j}^{n}\left(\frac{1}{1-p_{j}}\right) \times\left(\frac{S}{B_{j}}\right)
\end{aligned}
$$

where $S$ is the packet size, $p_{j}$ is the rate of packet loss and $B_{j}$ is the transmission rate of link $j$. The main idea behind the design of ETT metric is the use of multi radios in multi hop wireless networks to enhance the network performance. ETT is the amalgamation of packet loss rate and transmission rate of each individual link. ETT is an enhanced version of ETX with improved performance but still inherit the drawbacks of ETX being unaware of traffic load, intra-flow interference, inter-flow interference and channel diversity in MCMR WMNs. The design of ETT does not capture the losses due to contention caused by the traffic generated by the neighboring nodes. The traffic generated from the neighboring nodes contribute in the losses in two ways. First, it causes increase in collision which definitely increases the packet loss ratio. Secondly, it consumes the channel bandwidth. Active probing mechanism implemented in the design of ETT to capture the transmission rate may lead to over estimation during the time when the communication channels are quite busy. Although minimum delay (MD) [53] and improved expected transmission time (iETT) [54] are delay based routing metrics, but both of them inherit the basic drawbacks of ETT.

\subsection{Weighted cumulative ETT}

Weighted cumulative ETT (WCETT) is designed for high throughput, multi-hop, and multi-radio wireless networks with static nodes. WCETT is an extension of ETT by considering the packet loss ratio and transmission rate of each link separately. ETT of individual links are combined together to calculate the WCETT [11]. It captures the interference between the links using the same communicating channel. WCETT is defined as follows:

$$
\text { WCETT }=(1-\beta) \sum_{i=1}^{n} \operatorname{ETT}_{i}+\beta \max _{1 \leq j \leq k} X_{j}
$$


where $n$ indicates the total number of nodes, hops or links on the path, $k$ is the total number of available channels for multi-radio interfaces, $\beta$ is a tunable parameter ranging between 0 and 1 and $X_{j}$ is defined as follows:

$$
X_{j}=\sum_{\text {hop } i \text { on channel } j} \operatorname{ETT}_{i} \text { where as } 1 \leq j \leq k
$$

$X_{j}$ indicates the summation of transmission times required by each hop on the channel $j$. Hence overall throughput of the path will be based on the bottleneck channels which have the highest value of $X_{j}$. Although $X_{j}$ leads WCETT to the paths having high channel diversity to reduce the intra-flow interference but it fail to capture the inter-flow interference and traffic loads. Thus it leads the traffic towards congested paths in the network. Furthermore WCETT is non isotonic in nature thus does not support loop free routing [37]. WCETT-load balancing (WCETT-LB) is an extension of WCETT with a load balancing component, it provides a mechanism for load distribution in the network to avoid congested route. It measures the traffic concentration and traffic congestion at node level on a particular path $p$ but again it fails to capture the inter-flow interference [55]. As a result, WCETT-LB does not perform efficiently in multi-radio networks and is explained as follows:

$$
\operatorname{WCETT}(\text { path })=\operatorname{WCETT}(\text { path })+L(\text { path })
$$

where $L$ (path) is a load balancing component of the path and is defined as follow:

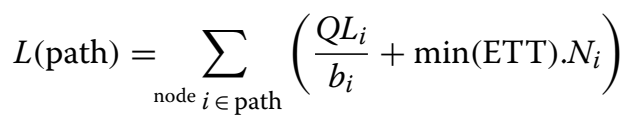

$Q L_{i}$ indicates the mean or average queue length and $b_{i}$ gives the transmission rate which captures the level of congestion at each node where as $N_{i}$ indicates the total number of child nodes using node $i$ as their next hop on path $p$ which actually predict the intensity of traffic congestion or concentration at each node.

\subsection{Metric of interference and channel switching}

WMNs have shared medium hence intra-flow interference, i.e., interference between nodes on the path of same flow and inter-flow interference, i.e., interference between the nodes, are very critical especially in MCMR networks. Due to interference, the load carrying capacity of the links is affected and the overall performance of the network is degraded. Metric of interference and channel switching (MIC) is developed to capture the interference aware parameters of the links [37]. It deals with both inter-flow and intra-flow interference to predict the quality of the path for efficient routing. Metric of interference and channel switching is based on ETT and is explained as follows:

$$
\mathrm{MIC}_{p}=\frac{1}{N \times \min (\mathrm{ETT})} \sum_{\text {link } l \in p} \mathrm{IRU}_{l}+\sum_{i \in p} \mathrm{CSC}_{i}
$$

and

$$
\mathrm{IRU}_{l}=\mathrm{ETT}_{l}+N_{l}
$$

where IRU indicates interference aware resource usage that predicts inter-flow interference on the basis of ETT which is the minimum ETT available in the network measured with the help of minimum transmission rate of the interference card, channel switching cost (CSC) that predicts the intra-flow interference and $\mathrm{N}$ is the number of neighboring nodes interfered by the link $l$ on the path $p$. $\mathrm{CSC}_{i}$ is defined as follows:

$$
\begin{aligned}
& \mathrm{CSC}_{i}=\left\{\begin{array}{ll}
w 1 & \text { if } \mathrm{CH}(\operatorname{prev}(\mathrm{i})) \neq \mathrm{CH}(\mathrm{i}) \\
w 2 & \text { if } \mathrm{CH}(\operatorname{prev}(\mathrm{i}))=\mathrm{CH}(\mathrm{i})
\end{array}\right\} \\
& 1 \leq w 1 \leq w 2
\end{aligned}
$$

where $\mathrm{CSC}_{i}$ indicates the channel reserved for node $i^{\prime} s$ transmission and prev $(i)$ denotes the previous hop of the node $i$ through the route $p$. Thus CSC can capture the inference only between two successive links. MIC extends ETT by considering the intra and inter-flow interference required in MCMR WMNs but still lacks in load balancing and isotonic characteristics. To make MIC isotonic in nature, decomposition is carried out by transforming the real network into virtual networks which further increases its complexity [56,57]. Moreover, it only measures the interference in a static way which is actually the total number of interfering node that may or may not be causing interference at that time. Thus MIC prefers nodes having less number of neighbors, as a result of which traffic will be routed towards the edges or the boundary of the network [58]. MIC required dynamic information about ETT of each link in the network which introduces the overhead and degrade the performance efficiency of the network.

\subsection{Load aware expected transmission time}

Load aware expected transmission time (LAETT) has incorporate the load balancing and link quality component in ETT to remove the drawback of ETT [59]. Load aware ETT is a combination of ETT and remaining capacity (RC) on the node. RC of link is used as load aware parameter to balance the traffic on the network. If two paths have same value of ETX then LAETT will prefer the paths having high value of RC. Loop free route or isotonic nature of 
the LAETT is due to the fact that it calculates the weights on each link. RC is designed to capture the total loads on the links and is defined as follows:

$$
\mathrm{RC}_{j}=B_{j}-\sum_{\text {flow }} \in \in N_{j}\left(f_{j k} \cdot \gamma_{j k}\right)
$$

where $f_{j k}$ represents the transmission rates of the $N_{j}$ flows, i.e., total number of current flows passing through node $j$, $B_{j}$ indicates the transmission rate of node $j$ and link quality factor of node $j$ is represented by $\gamma_{j k}$. LAETT is defined as follows:

$$
\operatorname{LAETT}_{i j}=\operatorname{ETX}_{i j} \times \frac{S}{\left(\frac{\mathrm{RC}_{i}+\mathrm{RC}_{j}}{2 \cdot \gamma_{i j}}\right)}
$$

where as $\gamma_{i j}$ is a link quality factor, $\mathrm{RC}_{i}$ and $\mathrm{RC}_{j}$ are the $\mathrm{RC}$ of the node $i$ and $j$, respectively. Practically $\mathrm{RC}$ is calculated at layer 2 by measuring the free slots and completed slots provided by the modulation scheme in use. Transmission rate measurements in LAETT are carried out with the help of total number of flows passing across the node and are assumed to be of same data rate. This is actually not true in relation with the wireless networks as the data rates vary because of congestion and interference over the links from time to time. Moreover, different radios and applications utilizing the network have different transmission rate. Probing mechanism used in the design of LAETT to measure ETX may result in underestimation of the link quality. Furthermore, Equation (15) does not predict any information about the intra-flow and inter-flow interference, which is very critical in MCMR environments.

\subsection{Airtime cost routing metric}

IEEE $802.11 \mathrm{~s}$ is an amendment of standard IEEE 802.11 for WMNs where as Airtime Cost Routing metric is the default routing metric defined in IEEE 802.11s [30]. It is an interface aware (iAWARE) routing metric developed for communication between different IEEE 802.11s standards. Airtime cost captures the information related to the channel utilization during transmission, e.g., transmission rate, overhead, and frame error rate. It measures the load on each relay node in terms of mean delay faced by the transmission of packets having size equal to $1 \mathrm{~kb}$ [60]. Airtime channel cost of a link is calculated as follows:

$$
C_{a}=\left[O_{c a}+O_{p}+\frac{B_{t}}{r}\right] \times \frac{1}{1-e_{p t}}
$$

where $O_{c a}$ represents channel access constant, $O_{p}$ indicates protocol overhead, $B_{t}$ is the number of bits in the test frame, $r$ is the node transmission rate in Mbit per second and $e_{p t}$ frame error rate for the test frame having size equal to $B_{t}$. The taxonomy of Airtime Cost routing shows that, it is very close to ETT. Actually $\left(O_{c a}+O_{p}+B_{t} / r\right)$ indicates the transmission time and $\left(1 / 1-e_{p t}\right)$ indicates the number of retransmissions same as ETT. No load balancing mechanism is defined in this metric which may lead the route to congested areas. Airtime metric is unaware of intra-flow interference which has a significant effect on the network performance in MCMR WMNs. Moreover, active probing mechanism to capture the data rate and losses cause overhead in the network depending on the traffic congestion. Therefore, airtime cost metric does not predict the actual quality of the link [61].

\subsection{Interference aware routing metric}

iAWARE routing metric addresses the problem of interference in MCMR WMNs by combining the interference ratio (IR) with the ETT metric. Predication of the link quality is based on the measurement carried out with the help of variation in link loss ratio and transmission rate parameters. Physical interference model is used to predict the interference faced by the links over the network using ratio between SINR (Signal to Noise Plus Interference Ratio) and SNR (Signal-to-Noise Ratio) at each node $[58,62]$. iAWARE is defined as follows:

$$
\text { iAWARE }=(1-\alpha) \sum_{i=1}^{n} \operatorname{iAWARE}_{i}+\alpha \max _{1 \leq j \leq k} X_{j}
$$

where iAWARE measured the inter-flow interference in the network, $X_{j}$ predicts the channel diversity and route towards the less intra-flow interference areas, $k$ is the total number of channels, $n$ is total number of links and $p$ indicates the path of the network. $\alpha$ is defined as the trade off parameter to tune between intra-flow and inter-flow interferences of the path. Inter-flow component of interference of the link is calculated as follows:

$$
\text { iAWARE }_{i}=\frac{E T T_{i}}{I R_{i}}
$$

where $\mathrm{IR}_{i}$ is the interference ratio of the link $i$ and is defined as follows:

$$
\mathrm{IR}_{i}=\frac{\mathrm{SINR}_{i}}{\mathrm{SNR}_{i}}
$$

$\operatorname{SINR}_{i}$ and $\mathrm{SNR}_{i}$ are calculated by the following equations:

$$
\begin{aligned}
\operatorname{SINR}_{i} & =\frac{P_{i}}{N} \\
\mathrm{SNR}_{i} & =\frac{P_{i}}{N+\sum_{w \in N_{i}-v} \cdot \tau_{w} \cdot P_{w}}
\end{aligned}
$$

where as $P$ indicates the signal strength, $N$ is the background noise and $\tau_{w}$ is the fraction of time period for 
which node $w$ makes the channel busy. $X_{j}$ in Equation (17) can be calculated as follows:

$$
X_{j}=\sum_{\text {conflicting links } i \text { on channel } j} \text { iAWARE }_{i}
$$

where $1 \leq j \leq k$.

Basically iAWARE is non isotonic in nature like WCETT thus cannot be used in link state routing protocols, i.e., OLSR. It only predicts the interference on the receiver side where as sender side interference component is also important for quality routes. Moreover, iAWARE has no MAC layer interference measurement mechanism, as it only capture the interference at a node level in terms of ratio between SINR and signal strength $P$ which is being received from other interfering nodes [56]. Lack of load balancing parameters may lead the traffic to congested route. When the value $\mathrm{IR}_{i}$ of the link is greater then $\mathrm{ETT}_{i}$ in Equation (18) then the value of $\mathrm{iAWARE}_{i}$ becomes small causing the traffic to route towards the links having small value of ETT but may have higher level of interference causing performance degradation in the network.

\subsection{Interferer neighbors count routing metric}

Interferer neighbors count routing metric (INX) is a radio aware routing metric which actually the improved version of ETX by considering the interference parameters to optimize the radio resource utilization cost [63]. INX of the link is calculated as follows:

$$
\mathrm{INX}_{j}=\mathrm{ETX}_{j} \cdot \sum_{k \in N_{j}} r_{k}
$$

where $N_{j}$ indicates the number of interfering links resulting from the transmission taking place on link $j, r_{k}$ represents the available transmission rate of the link $k$. Although INX is isotonic in nature, it performs well only under low load scenarios because no load balancing mechanism is defined in the routing metric. As a result, it faces quick performance degradation as the network load increases. Moreover, it uses a probing technique to measure the interference parameters of the link in a static way which causes an overhead and also fail to predict the true quality of the link. Nevertheless INX behave in a better way as compared to MIC because it follows asymmetric links and isotonic behavior [42].

\subsection{Resource aware routing for MESH}

Resource aware routing metric (RARE) has a passive monitoring technique to capture the radio link quality parameters related to load and interference in order to overcome the overhead caused by active probing mechanism [64].
RARE is the combination of bandwidth, contention and signal strength and is defined by the following equation:

$$
\operatorname{RARE}_{i}=\alpha \cdot \frac{C-B W_{a}}{B W_{a}}+\beta \cdot \frac{\mathrm{RSSI}_{\text {max }}-\mathrm{RSSI}}{\mathrm{RSSI}}+\gamma \cdot N_{c}
$$

where $\mathrm{BW}_{a}$ is the available bandwidth, RSSI is the RSSI value, $\mathrm{RSSI}_{\max }$ is the maximum value or RSSI, $C$ is the link capacity, $N_{c}$ is the average contention and $\alpha, \beta$ and $\gamma$ are weights associated with bandwidth, RSSI, and contention components, respectively. Available bandwidth component $\mathrm{BW}_{a}$ is further defined as follows:

$$
\mathrm{BW}_{a}=\left(\frac{T_{\text {idle }}}{T_{\text {busy }}+T_{\text {idle }}}\right) \cdot T X_{\text {rate }}
$$

where $T X_{\text {rate }}$ is the transmission rate, $T_{\text {idle }}$ is the idle time interval and $T_{\text {busy }}$ is the busy time interval, respectively, for the calculation of traffic load based on passive monitoring technique. Low overhead RARE can predict the inter-flow interference through contention component $N_{c}$ as defined in Equation (24) in a passive manner but fail to predict the intra-flow interference and channel diversity in MCMR WMNs. Moreover, passive measurements does not predict about the brusty losses which normally occur in wireless links. As a result RARE may under estimate the link quality of the network. Furthermore, WMNs use Common-off-The-Shelf (COTS) products so normally their network cards or drivers do not support passive monitoring while transmitting which may result in the performance degradation.

\subsection{Contention aware transmission time}

Contention aware transmission time (CATT) routing metric is a load aware and iAWARE routing metric which is actually based on ETT [56]. Key functions of CATT is that it predicts location dependent contention and rate diversity of the links. Isotonic behavior of CATT makes it possible to work with link state routing protocols. CATT is calculated as follows:

$$
\operatorname{CATT}_{i}=\operatorname{ETX}_{i} \cdot \sum_{j \in N_{i}}\left(\left(\sum_{K \in N_{j}} \frac{R_{k}}{L_{k}}\right) \cdot \tau_{j} \cdot \frac{R_{j}}{L_{j}}\right)
$$

where $N_{i}$ is total number of links interfering the transmission taking place on the link $i$. Similarly $N_{j}$ is total number of links interfering the transmission taking place on the link $j . R_{k}$ and $R_{j}$ indicate the packet size of the links containing 1 and 2 hop neighbors, respectively. $B_{k} \& B_{j}$ measure the bandwidth of links in 1 and 2 hop neighbors, respectively. $\tau_{j}$ is defined as packet transmission attempt rate on link $j$. 
Although CATT captures the inter-flow and intra-flow interferences simultaneously [59]. Like MIC, CATT also assumes that all the neighboring nodes are participating in the inference parameters (weather or not they are involved in transmitting the data) which may overestimate the link quality. Another important drawback in CATT is that it uses active probing mechanism to measure the interference and delay which causes large overhead in the network. Hence this metric is not suitable for triple play application networks where the traffic is quite congested [65]. Moreover, delay in transmission is used to measure the traffic load which does not predict the load in an accurate way.

\subsection{Interference load aware routing metric}

The interference load aware (ILA) is a hybrid metric based on the load, interfering neighbors, and transmission rates, specially designed for multi-channel WMNs [66]. Path weight of the metric is defined as follows:

$$
\mathrm{ILA}_{p}=\alpha \times \sum_{\text {link } i \in p} \mathrm{MTI}_{i}+\sum_{\text {node } i \in p} \mathrm{CSC}_{i}
$$

where $p$ is the path in the network, metric of traffic interference (MTI) and CSC which measures the efficiency of flows routed through the path $p$. These two components of the metric measure the intra flow interference, inter flow interference, transmission rates, congested areas, and packet loss ratios.

MTI is the first component which measures the quantity of traffic generated by the interfering neighboring nodes instead of number of interfering neighboring nodes as defined in MIC. MTI is defined as follows:

$$
\operatorname{MTI}_{i}(C)=\left\{\begin{array}{ll}
\operatorname{ETT}_{i j}(C) \times \operatorname{AIL}_{i j}(C), & N_{i} \neq 0 \\
\operatorname{ETT}_{i j}(C), & N_{i}=0
\end{array}\right\}
$$

where ETT measures the difference in transmission rate and packet loss ratio of the links in the Network. When node $i$ and node $j$ are transmitting over channel $C$, average interfering load (AIL) is defined as follows:

$$
\mathrm{AIL}_{i j}(C)=\frac{\sum_{N_{l}} \mathrm{IL}_{i j}(C)}{N_{l}(C)}
$$

where $N_{l}(C)$ is the set of interfering neighbor of the node $i$ and $j$ and is defined as follows:

$$
N_{l}(C)=N_{i} \cup N_{j}
$$

where $I L_{i j}(C)$ is the interference load of the neighbors.

CSC which is the second component of ILA, captures the intra-flow interference and is same as defined in
Equation (12). $\alpha$ which is a scaling factor to balance the effect of MTI and CSC is defined as follows:

$$
1 / \alpha=\left\{\begin{array}{cc}
\min (\mathrm{ETT}) \cdot \min (\mathrm{AIL}), & N_{i} \neq 0 \\
\min (\mathrm{ETT}), & N_{i}=0
\end{array}\right\}
$$

where $\min (\mathrm{ETT})$ and $\min (\mathrm{AIL})$ is the smallest ETT and average load in the network, respectively. In order to capture the difference in transmission rate, packet loss ratio, intra-flow interference, and inter-flow interference. ILA uses a active probing mechanism which induces a large overhead in the network. However, it may not be suitable for congested traffic areas. Since as it is based on ETX and ETT, it inherits their drawbacks. Exposed node terminal problem causes the interference to occur in two hop range instead of one hop range as consider in ILA and MIC, results in the underestimation of the link quality. Furthermore, ILA does not consider the transmission delay in order to route the traffic efficiently [67].

\subsection{Contention window based routing metric}

Contention window based $(\mathrm{CWB})$ routing metric routes the traffic by considering the channel utilization and average contention window used on the links. CWB is a load-interference aware routing metric which guides the routing protocol to balance the traffic load on the links and to increase the network throughput by routing towards less congested traffic areas [40]. The congestion level and channel utilization at a particular node of a network is given as follows:

$$
\mathrm{CWB}=\beta \cdot \overline{\mathrm{CW}}
$$

Congestion level is measured by the average value of contention window on link $\overline{\mathrm{CW}}$ and is further defined by the following equation [68]:

$$
\mathrm{CW}=\frac{1-\mathrm{FER}}{1-\mathrm{FER}^{r+1}}+\frac{1-(2 . \mathrm{FER})^{r+1}}{1-(2 . \mathrm{FER})} \mathrm{CW}_{0}
$$

where FER is the measure of frame error rate, $\mathrm{CW}_{0}$ is the measure of minimum contention window and $r$ capture the maximum back off stage. Channel utilization component $\beta$ represents the channel busy time CBT, i.e., amount of time that a channel spends in transmitting, receiving, and occupying states. $\beta$ is defined as follows:

$$
\beta=\left\{\begin{array}{cc}
1, & \text { if } u \leq T \\
\min \left(\alpha \cdot\left(u-T_{1}\right)+\exp \left(\frac{u-T_{1}}{T_{2}-u}\right), \beta_{\max }\right), & \text { if } T_{1}<u<T_{2} \\
\beta_{\max }, & \text { if } u \geq T_{2}
\end{array}\right\}
$$

So $\beta$ is equal to 1 when channel utilization is quite small and $\beta$ is equal to $\beta_{\max }$ when channel utilization is maximum. Where as $T_{1}$ and $T_{2}$ indicate the minimum and maximum threshold values of the channel utilization represented by $u$ and the value $\alpha$ will decide about the 
change in the value of $\beta$ as channel utilization $u$ passes the threshold value $T_{1}$. CWB can only capture the inter-flow interference and traffic load but fail to capture the intraflow interference which is a critical parameter for MCMR WMNs. Furthermore, this metric perform poor when the network conditions change quickly because calculations needed to find out the size of $\mathrm{CW}$ are quite sophisticated [42].

\subsection{Metric for interference and channel diversity}

Metric of interference and channel diversity (MIND) captures interference and load aware parameters on the basis of passive monitoring technique thus reducing the overhead usually caused by active probing mechanism to support internet traffic and many other applications like VoIP and video streaming in multi casting and peer-to-peer service models [69] and is defined as follows:

$$
\text { MIND }=\sum_{\text {link }}^{n} \operatorname{INTERLOAD~}_{i}+\sum_{\text {node }}^{m \in p} C_{j}^{m} C S C_{j}
$$

where INTERLOAD component captures the inter-flow interference and load, CSC capture the intra-flow inference and is same as calculated in Equation (12), $n$ measures the total number of wireless links, $m$ indicates the total number of nodes over the path $p$. The INTERLOAD component is defined as follows:

$$
\operatorname{INTERLOAD}_{j}=\left(\left(1-I R_{j}\right) \cdot \tau\right) \cdot C B T_{j}
$$

where $0 \leq R I \leq 1$ and $0 \leq C B T \leq 1$.

IR is the interference ratio and is used to measure the interference between the links and is calculated as follows:

$$
\mathrm{IR}_{j}=\frac{\mathrm{SINR}_{j}}{\mathrm{SNR}_{j}}
$$

MIND uses a passive mechanism to capture the single strength values in Equation (37) through wireless cards. Channel busy time (CBT) is also calculated passively to predict load by using the following equation:

$$
\mathrm{CBT}_{j}=\frac{\text { Total time }- \text { Idle time }}{\text { Total time }}
$$

Total Time is the measure of time between the first attempt to send the packet and the reception of its acknowledge. Idle Time is the measure of back off times and the time in which the radio nodes sense that the medium is free for access. Thus CBT is the measure of time spend during transmission, receiving, and occupying states.

Overall, MIND captures the inter-flow interference and intra-flow interference in intelligent manner by considering physical and logical interference models. The major limitation of MIND is its non isotonic nature which induced complexity in its implementation through virtual networks. IR component of MIND is quite different from the IR designed for iARWE because the design principle of MIND focus on the node parameters where as design of iARWE is based on link parameters. Furthermore, MIND does not judge the asymmetry of the links which cause erroneousness in channel quality measurement parameters. MIND considers back off time period as an idle time. Therefore, it may underestimate the channel interference. Unlike MIND, interference-delay aware routing metric for multi-interface mesh networks balances the load by using multi-interface multi-channel capabilities of the node but the interference is measured in a static manner which actually underestimate the quality of link in the network [67]. Similarly channel utilization and contention window based (C2WB) metric is a interference and load aware metric which capture the inter-flow component of interference and congestion but unaware of the intraflow component of interference and channel diversity in multi-interface WMNs [70].

\section{Summary}

Routing metrics play a critical role in path selection and in route optimization in MCMR WMNs. Routing metrics are composed of set of parameters capture at different layers of the OSI model to predict about the quality of the link, e.g., path length, delay, packet loss ratio, queue size, link capacity, and interference. On the basis of these parameters routing metrics can be classified as simple, interference aware, load aware, and load \& interference aware. Figure 6 shows a timeline framework of routing metrics in which publication dates of each routing metric were used to build the timeline diagram. Moreover, arrows are used to represent the dependency between the predecessor routing metrics and the potentially influenced routing metrics. Qualitative comparison of these routing metrics in terms of their characteristic and limitations are summarized in Figure 7.

Simple routing metrics for WMNs utilize transmission rate, packet loss ratio, and delay parameters to capture the link quality of the link, e.g., ETX, mETX, ETT, ENT, $\mathrm{MD}$, and iETT. These routing metrics are simple in design and easy to implement in the routing protocol but they lack in capturing the load and interference aware parameters of the links. Furthermore, these routing metrics are unaware of channel diversity in MCMR scenarios. As a result, these routing metrics lead the traffic towards the congested areas. Hence not suitable for efficient routing in MCMR WMNs.

Interference aware routing metrics capture the interflow and intra-flow interference parameters along with the transmission rate and packet loss ratio to predict the quality of the link. Thus increasing the intelligence behavior of the cross layer routing metrics as interference has a critical 


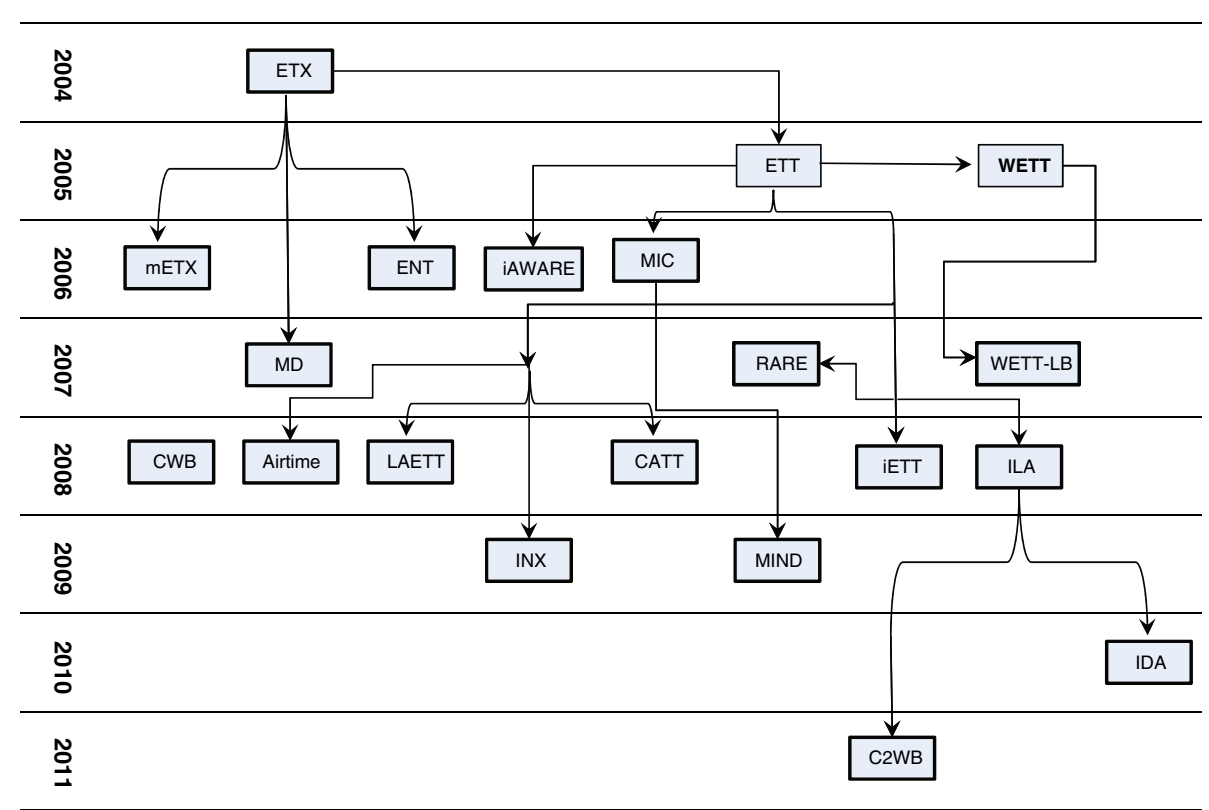

Figure 6 Cross layer routing metrics with timeline and potential influence.

effect on the delay and overall throughput of the network in MCMR WMNs. WCETT, MIC, iAWARE, INX and Airtime Cost are the examples of iAWARE routing metrics. Although theses routing metrics perform quiet efficiently as compared to ETX and ENT but still lack in load balancing features. Furthermore, some of them only capture the single component of interference, i.e., inter-flow or intraflow interference although both are mandatory for quality links in real time applications. Non isotonic behavior of some of these routing metrics make their implementation in the routing protocol quite complex because they demand virtual networks to produce loop free routing.

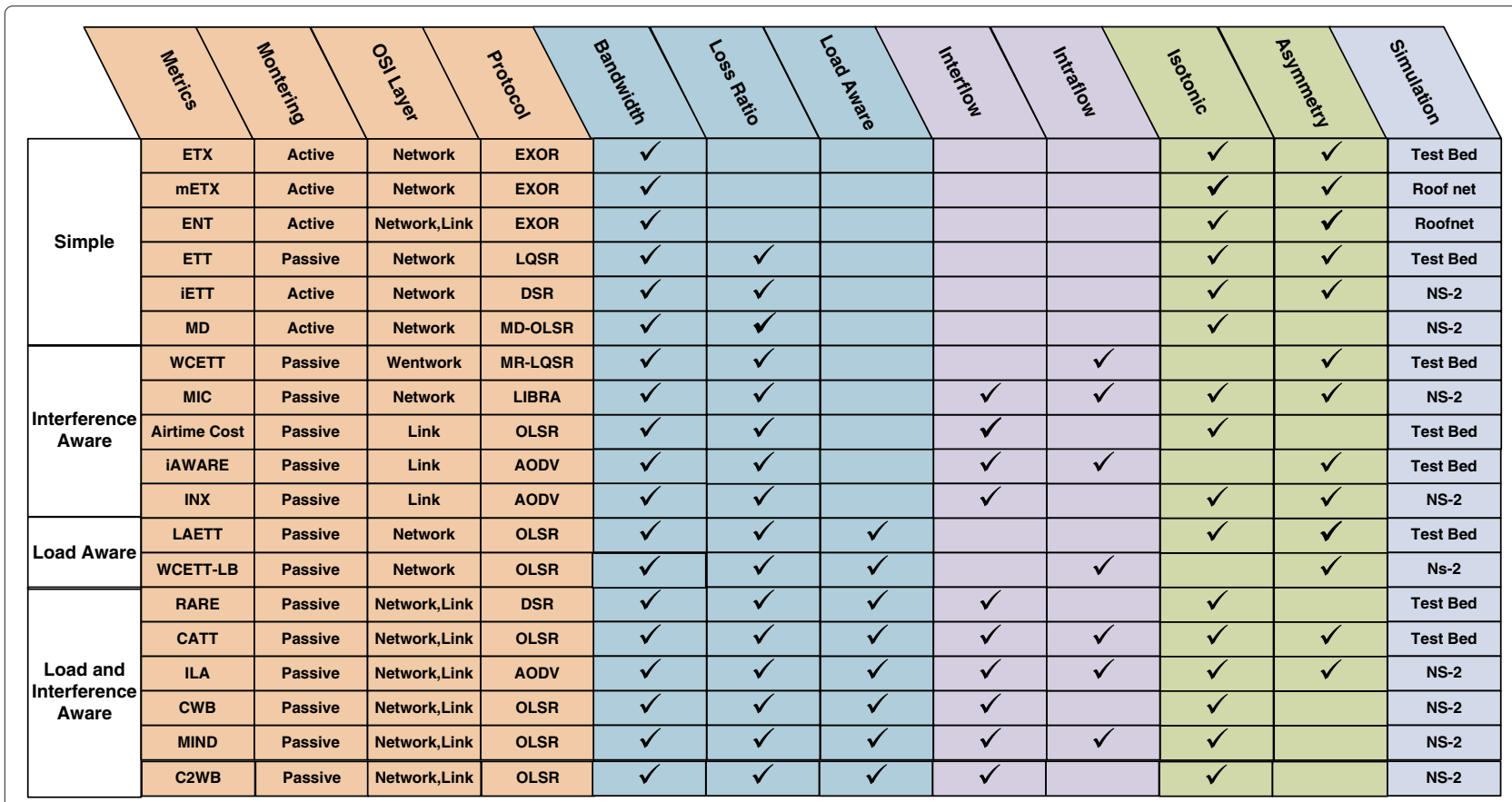

Figure 7 Qualitative comparison of cross layer routing metrics in MCMR WMNs. 
Load aware routing metrics capture the traffic concentration and congestion parameters at node level to introduce load awareness in the routing which has a significant effect specially in multi casting and real time applications, i.e., VoIP and Video over IP in community networks. Examples of theses routing metrics are LAETT and WCETT-LB. Since they are based on EXT and ETT, so they inherit their drawback and make the traffic to route towards boundaries of the network. Moreover, they are unaware of inter-flow interference in MCMR WMNs.

Load $\mathcal{E}$ interference aware routing metrics, e.g., RARE, CATT. ILA, CWB, MIND, and C2WB are most recent development in routing metrics as they incorporate the transmission rate, packet loss ratio, congestion, channel diversity, and interference parameters in to the quality aware cross layer routing metric for MCMR WMNs. They actually interrelate traffic load and interference in the network and lead the network traffic towards efficient routes. The key benefit of MIND is that it uses a passive monitoring technique to overcome the overhead caused by active monitoring. Moreover, it does not inherit the drawbacks of ETX or ETT as it is not based on them.

In spite of these routing metrics, cross layer routing metric design is still an open research issue in MCMR WMNs for QoS specially in real time applications.

\section{Conclusions}

In this article, we provide a comprehensive taxonomy and qualitative comparison of most recent cross layer routing metrics in MCMR WMNs with respect to their design factors, characteristics and limitations. Study revealed that load \& interference aware cross layer routing metrics are more efficient to pick up link quality parameters as compared to simple, interference, and load aware routing metrics in highly congested traffic areas especially in real time applications like VoIP and Video over IP in multi casting and peer-to-peer service models. Moreover, this research open up several future investigations regarding cross layer design of routing metrics in terms of load balancing and route stability mechanism in MCMR WMNs. Logical and Physical models for interflow and intra-flow interference measurements need to be further investigated. Furthermore, integration of WMNs with other network technologies like WiMAX, MANETS, VANETS, Wireless Sensor Networks (WSN) and cellular networks, need to be further investigated.

\section{Competing interests}

The authors declare that they have no competing interests.

\section{Author details}

${ }^{1}$ Faculty of Computer Science and Information Systems, Universiti Teknologi Malaysia, 81310 Skudai, Johor, Malaysia. ${ }^{2}$ Faculty of Computer Science and Information Technology, University of Malaya, 50603 Lembah Pantai, Kuala Lumpur, Malaysia.
Received: 21 December 2011 Accepted: 30 March 2012

Published: 21 May 2012

\section{References}

1. J Ishmael, S Bury, D Pezaros, Race, N Deploying rural community wireless mesh networks. IEEE Internet Comput. 12(4), 22-29 (2008)

2. IF Akyildiz, X Wang, W Wang, Wireless mesh networks: a survey. Comput. Netw. 47(4), 445-487 (2005). http://www.sciencedirect.com/science/ article/B6VRG-4F53V5H-2/2/9fa1587e47665f1fb3f7fb461461dd6b

3. H Zhu, M Li, I Chlamtac, B Prabhakaran, A survey of quality of service in IEEE 802.11 networks. IEEE Wirel. Commun. 11(4), 6-14 (2004)

4. M Mauve, A Widmer, H Hartenstein, A survey on position-based routing in mobile ad hoc networks. IEEE Netw. 15(6), 30-39 (2001)

5. I Akyildiz, W Su, Y Sankarasubramaniam, E Cayirci, A survey on sensor networks. IEEE Commun. Mag. 40(8), 102-114 (2002)

6. C Lochert, H Hartenstein, J Tian, H Fussler, D Hermann, M Mauve, in IEEE Proceedings on Intelligent Vehicles Symposium, vol. 9-11. A routing strategy for vehicular ad hoc networks in city environments, (Ohio, USA, June 2003), pp. 156-161

7. B Li, Y Qin, CP Low, CL Gwee, A survey on mobile WiMAX [wireless broadband access]. IEEE Commun. Mag. 45(12), 70-75 (2007)

8. IF Akyildiz, X Wang, A survey on wireless mesh networks. IEEE Commun. Mag. 43(9), S23-S30 (2005)

9. X Wang, AO Lim, IEEE 802.11s wireless mesh networks: Framework and challenges. Ad Hoc Netw. 6(6), 970-984 (2008). http://www.sciencedirect. com/science/article/B7576-4PYGW06-1/2/ 1e8e8e83a5dcfe4e4b696af4427ab690

10. S Seth, A Gankotiya, A Jindal, in 2010 Second International Conference on Computer Engineering and Applications (ICCEA), vol. 1. Current state of art research issues and challenges in wireless mesh networks, (Bali Island, Indonesia, 19-21 March 2010), pp. 199-203

11. R Draves, J Padhye, B Zill, in Proceedings of the 10th annual international conference on Mobile computing and networking. Routing in multi-radio, multi-hop wireless mesh networks (MobiCom'04, ACM, New York, 2004), pp. 114-128. http://doi.acm.org/10.1145/1023720.1023732

12. J Yuan, Z Li, W Yu, B Li, A cross-layer optimization framework for multihop multicast in wireless mesh networks. IEEE J. Sel. Areas Commun. 24(11), 2092-2103 (2006)

13. Campista EPMICLDOPDdACSDRM MEM, IEEE Netw. 22, 6-12 (2008). http://www.scopus.com/inward/record.url?eid=2-s2.0-39549084697\& partnerlD=40\&md5=d5cc6a26b37fe6da6b37ea6fd06e5625

14. R Bruno, M Conti, E Gregori, Mesh networks: commodity multihop ad hoc networks. IEEE Commun. Mag. 43(3), 123-131 (2005)

15. RP Karrer, A Pescape, THuehn, Challenges in second-generation wireless mesh networks. EURASIP J Wirel Commun Netw. 2008, 274790 (2008)

16. B Manoj, R Ananthapadmanabha, CSR Murthy, Multi-hop cellular networks: Architecture and protocols for best-effort and real-time communication. J. Parallel Distrib. Comput. 65(6), 767-791 (2005). http:// www.sciencedirect.com/science/article/B6WKJ-4FVH475-1/2/ b6d4e85d0cd2fccb59385e024a0bf5ae

17. S Waharte, R Boutaba, Y Iraqi, B Ishibashi, Routing protocols in wireless mesh networks: challenges and design considerations. Multimedia Tools Appl. 29, 285-303 (2006). http://dx.doi.org/10.1007/s11042-006-0012-8

18. A Raniwala, T-c Chiueh, in Proceedings IEEE INFOCOM 2005. 24th Annual Joint Conference of the IEEE Computer and Communications Societies, vol. 3. Architecture and algorithms for an IEEE 802.11-based multi-channel wireless mesh network, (Miami, USA, 13-17 March 2005), pp. 2223-2234

19. S Avallone, IF Akyildiz, A channel assignment algorithm for multi-radio wireless mesh networks. Comput. Commun. 31(7), 1343-1353 (2008). http://www.sciencedirect.com/science/article/B6TYP-4RP0MMN-N/2/ 4bbdb4664362311783a2ceedfcb37610

20. F Licandro, G Schembra, Wireless Mesh networks to support video surveillance: architecture, protocol, and implementation issues. EURASIP J Wirel Commun Netw. 2007, 031976 (2007)

21. K Sethom, T Ali-Yahiya, N Laga, G Pujolle, A QoS-aware mesh protocol for future home networks using autonomic architecture. EURASIP J Wirel Commun Netw. 2008, 386898 (2008)

22. M Sichitiu, Wireless mesh networks: opportunities and challenges. EURASIP J. Wirel. Commun. Net, 318-323 (2005). http://www.scopus. com/inward/record.url?eid=2-s2.0-20444444697\&partnerlD=40\&md5= ce4c45f7608078b123b4c8e647af428b 
23. P Jacquet, P Mühlethaler, T Clausen, A Laouiti, A Qayyum, L Viennot, in IEEE INMIC'01. Optimized link state routing protocol (IEEE, Lahore, 28-30 December 2001), pp. 62-68. http://hipercom.inria.fr/olsr/ inmic2001.ps

24. AB Paul, S Nandi, in International Conference on Information Technology. Modified optimized link state routing (M-OLSR) for wireless mesh networks, (Bhubaneswar, India, 17-20 December 2008), pp. 147-152

25. CE Perkins, P Bhagwat, Highly dynamic destination-sequenced distance-vector routing (DSDV) for mobile computers. SIGCOMM Comput. Commun. Rev. 24, 234-244 (1994). http://doi.acm.org/10.1145/ 190809.190336

26. P King, A Etorban, IS Ibrahim, in 8th Annual PostGraduate Symposium on the Convergence of Telecommunications, Networking and Broadcasting. A DSDV-based multipath routing protocol for mobile ad-hoc networks, (Liverpool John Moores University, UK, 28-29 June 2007), pp. 93-98

27. C Perkins, E Royer, in Second IEEE Proceedings Workshop on Mobile Computing Systems and Applications, 1999. WMCSA'99. Ad-hoc on-demand distance vector routing, (New Orleans, Louisiana, USA, 25-26 February 1999), pp. 90-100

28. EM Royer, CE Perkins, in Proceedings of the 5th annual ACM/IEEE international conference on Mobile computing and networking. Multicast operation of the ad-hoc on-demand distance vector routing protocol (MobiCom'99, ACM, New York, 1999), pp. 207-218. http://doi.acm.org/10. $1145 / 313451.313538$

29. DB Johnson, DA Maltz, in Mobile Computing, The International Series in Engineering and Computer Science, vol. 353, ed. by TImielinski and HF Korth. Dynamic source routing in ad hoc wireless networks, (Springer, US, 1996), pp. 153-181. http://dx.doi.org/10.1007/978-0-585-29603-6_5

30. M Bahr, in IEEE Internatonal Conference on Mobile Adhoc and Sensor Systems MASS. Update on the hybrid wireless mesh protocol of IEEE 802.11s, (Pisa, Italy, 8-11 October 2007), pp. 1-6

31. VD Park, MS Corson, in Annual Joint Conference of the IEEE Computer and Communications Societies, vol. 3. A highly adaptive distributed routing algorithm for mobile wireless networks, (Kobe, Japan, 7-12 Apr 1997), pp. $1405-1413$

32. I Akyildiz, X Wang, Cross-layer design in wireless mesh networks. IEEE Trans.Veh. Technol. 57(2), 1061-1076 (2008)

33. L lannone, R Khalili, K Salamatian, S Fdida, in 1st International Symposium on Wireless Communication Systems. Cross-layer routing in wireless mesh networks, (Mauritius, 20-22 September 2004), pp. 319-323

34. A Pirzada, M Portmann, J Indulska, vol. 41, (2009), pp. 65-84

35. V Srivastava, M Motani, Cross-layer design: a survey and the road ahead. IEEE Commun. Mag. 43(12), 112-119 (2005)

36. K Jain, J Padhye, VN Padmanabhan, L Qiu, Impact of interference on multi-hop wireless network performance. Wireless Networks. 11, 471-487 (2005)

37. Y Yang, J Wang, R Kravets, Interference-aware load balancing for multihop wireless networks, Tech. rep. (University of Illinois at Urbana-Champaign, 2005)

38. N Bisnik, A Abouzeid, in IEEE International Conference on Communications, 2006. ICC'06, vol. 1. Delay and throughput in random access wireless mesh networks, (Istanbul, Turkey, 11-15 June 2006), pp. 403-408

39. G Wu, Chiueh Tc, in Proceedings of the 10th ACM Symposium on Modeling, analysis, and simulation of wireless and mobile systems. Passive and accurate traffic load estimation for infrastructure-mode wireless lan (MSWiM'07, ACM, New York, 2007), pp. 109-116. http://doi.acm.org/10. $1145 / 1298126.1298147$

40. LT Nguyen, R Beuran, Y Shinoda, in IEEE Symposium on Computers and Communications, ISCC. A load-aware routing metric for wireless mesh networks, (Marrakech, Morocco, 6-9 July 2008), pp. 429-435

41. TC Tsai, ST Tsai, A cross-layer routing design for multi-interface wireless mesh networks. EURASIP J Wirel Commun Netw. 2009, 4:1-4:8 (2009). http://dx.doi.org/10.1155/2009/208524

42. VC Borges, M Curado, E Monteiro, Cross-layer routing metrics for mesh networks: current status and research directions. Comput. Commun. 34(6), 681-703 (2011). http://www.sciencedirect.com/science/article/pii/ S0140366410004858

43. J Zhou, M Jacobsson, E Onur, I Niemegeers, An investigation of link quality assessment for mobile multi-hop and multi-rate wireless networks. Wirel. Pers Commun, 1-19 (2011). http://dx.doi.org/10.1007/s11277-011-0263-1
44. J Sobrinho, in INFOCOM 2001 IEEE Proceedings Twentieth Annual Joint Conference of the IEEE Computer and Communications Societies, vol. 2 Algebra and algorithms for QoS path computation and hop-by-hop routing in the Internet, (Anchorage, Alaska, USA, 22-26 April 2001), pp. $727-735$

45. D Kotz, C Newport, C Elliott. The mistaken axioms of wireless-network research, Tech. rep (Dartmouth College, 2003)

46. D Ganesan, B Krishnamachari, A Woo, D Culler, D Estrin, S Wicker, in Tech. rep. Complex behavior at scale: an experimental study of low-power wireless sensor networks (UCLA Computer Science Department, 2002). http://citeseerx.ist.psu.edu/viewdoc/summary?doi=10.1.1.19.770

47. K Ramachandran, I Sheriff, E Belding, K Almeroth, in INFOCOM 2001. IEEE Proceedings Twentieth Annual Joint Conference of the IEEE Computer and Communications Societies, vol. 3 of Lecture Notes in Computer Science. Routing stability in static wireless mesh networks (Springer, Berlin/Heidelberg, 2007), pp. 73-82

48. H Zhai, Y Fang, in Network Protocols, 2006. ICNP'06. 14th IEEE International Conference on Proceedings of the 2006. Impact of routing metrics on path capacity in multirate and multihop wireless ad hoc networks, (Santa Barbara, California, USA, 12-15 November 2006), pp. 86-95

49. DSJD Couto, D Aguayo, J Bicket, R Morris, A high-throughput path metric for multi-hop wireless routing. Wirel Netw. 11, 419-434 (2005). http://dx. doi.org/10.1007/s11276-005-1766-z

50. R Draves, J Padhye, B Zill, Comparison of routing metrics for static multi-hop wireless networks. SIGCOMM Comput. Commun. Rev. 34, 133-144 (2004). http://doi.acm.org/10.1145/1030194.1015483

51. C Koksal, H Balakrishnan, Quality-aware routing metrics for time-varying wireless mesh networks. IEEE J. Sel. Areas Commun.

24(11), 1984-1994 (2006)

52. B Sadeghi, V Kanodia, A Sabharwal, E Knightly, in Proceedings of the 8th annual international conference on Mobile computing and networking. Opportunistic media access for multirate ad hoc networks (MobiCom'02, ACM, New York, 2002), pp. 24-35. http://doi.acm.org/10.1145/570645. 570650

53. W Cordeiro, E Aguiar, W Moreira, A Abelem, M Stanton, in Proceedings of 16th International Conference on Computer Communications and Networks, 2007 ICCCN. Providing quality of service for mesh networks using link delay measurements, (Honolulu, Hawaii, USA, 13-16 August 2007), pp. 991-996

54. S Biaz, B Qi, Y Ji, in 5th IEEE Consumer Communications and Networking Conference, 2008. CCNC. Improving expected transmission time metric in multi-rate multi-hop networks, (Las Vegas, Nevada, USA, 10-12 January 2008), pp. 533-537

55. L Ma, M Denko, in 21st International Conference on Advanced Information Networking and Applications Workshops, 2007, AINAW'07, vol. 2. A routing metric for load-balancing in wireless mesh networks, (Niagara Falls, Ontario, Canada, 21-23 May 2007), pp. 409-414

56. M Genetzakis, V Siris, in 5th Annual IEEE Communications Society Conference on Sensor, Mesh and Ad Hoc Communications and Networks, 2008. SECON'08. A contention-aware routing metric for multi-rate multi-radio mesh networks, (San Francisco, California, USA, 16-20 June 2008), pp. 242-250

57. Y Yang, J Wang, R Kravets, Load-balanced routing for mesh networks. SIGMOBILE Mob. Comput. Commun. Rev. 10, 3-5 (2006). http://doi.acm. org/10.1145/1215976.1215979

58. A Subramanian, M Buddhikot, S Miller, in 2nd IEEE Workshop on Wireless Mesh Networks, 2006 WiMesh. Interference aware routing in multi-radio wireless mesh networks, (Reston, Virginia, USA, 25-28 September 2006), pp. 55-63

59. H Aiache, V Conan, L Lebrun, S Rousseau, in 5th IEEE International Conference on Mobile Ad Hoc and Sensor Systems, 2008. MASS. A load dependent metric for balancing Internet traffic in wireless mesh networks, (Atlanta, Georgia, USA, 29 September-2 October 2008), pp. 629-634

60. G Hiertz, D Denteneer, S Max, R Taori, J Cardona, L Berlemann, B Walke IEEE 802.11s: The WLAN Mesh Standard. IEEE Wirel. Commun. 17, 104-111 (2010)

61. M Islam, M Alam, M Hamid, C Hong, S Lee, EFT: a high throughput routing metric for IEEE 802.11s wireless mesh networks. Ann. Telecommun. 65, 247-262 (2010). http://dx.doi.org/10.1007/s12243-009-0130-1

62. P Gupta, P Kumar, The capacity of wireless networks. IEEE Trans. Inf. Theory. 46(2), 388-404 (2000)

63. R Langar, N Bouabdallah, R Boutaba, Mobility-aware clustering algorithms with interference constraints in wireless mesh networks. Comput. Netw. 
53, 25-44 (2009). http://www.sciencedirect.com/science/article/B6VRG4TK47J9-2/2/fd065ef52998750dec0d2edcc086b472

64. K Kowalik, B Keegan, M Davis, in IEEE International Conference on

Communications, 2007 ICC'07. RARE - Resource Aware Routing for mEsh, (Glasgow, Scotland, 24-28 June 2007), pp. 4931-4936

65. V Borges, M Curado, E Monteiro, in 2010 Proceedings of 19th International Conference on Computer Communications and Networks (ICCCN). A cross-layer routing scheme for scalable triple play service in wireless mesh networks, (Zurich, Switzerland, 2-5 August 2010), pp. 1-6

66. DM Shila, T Anjali, Load aware traffic engineering for mesh networks. Comput. Commun. 31(7), 1460-1469 (2008). http://www.sciencedirect. com/science/article/B6TYP-4RPOMMN-10/2/ $8 \mathrm{~d} 112 \mathrm{fe} 4 \mathrm{f3e} 25 \mathrm{~b} 28 \mathrm{e} 71 \mathrm{fb} 245 \mathrm{e} 8 \mathrm{f} 23 \mathrm{~b} 0 \mathrm{e}$

67. Q Tian, in 20106 th International Conference on Wireless Communications Networking and Mobile Computing (WiCOM). A new interference-delay aware routing metric for multi-interface wireless mesh networks, (Chengdu, China, 23-25 September 2010), pp. 1-5

68. R Beuran, LT Nguyen, K Latt, J Nakata, Y Shinoda, in 21st International Conference on Advanced Information Networking and Applications, 2007 AINA'07. QOMET: a versatile WLAN emulator, (Niagara Falls, Canada, 21-23 May 2007), pp. 348-353

69. V Borges, D Pereira, M Curado, E Monteiro, in Ad-Hoc, Mobile and Wireless Networks, vol. 5793, ed. by P Ruiz and I Garcia-Luna-Aceves. Routing metric for interference and channel diversity in multi-radio wireless mesh networks (Springer, Berlin Heidelberg, 2009), pp. 55-68

70. L Nguyen, R Beuran, Y Shinoda, An interference and load aware routing metric for wireless mesh networks. Int J Ad Hoc Ubiquitous Comput. 7, 25-37 (2011)

doi:10.1186/1687-1499-2012-177

Cite this article as: Bin Ngadi et al:: A taxonomy of cross layer routing metrics for wireless mesh networks. EURASIP Journal on Wireless Communications and Networking 2012 2012:177.

\section{Submit your manuscript to a SpringerOpen ${ }^{\circ}$ journal and benefit from:}

- Convenient online submission

- Rigorous peer review

- Immediate publication on acceptance

- Open access: articles freely available online

- High visibility within the field

Retaining the copyright to your article

Submit your next manuscript at $\boldsymbol{\wedge}$ springeropen.com 